\title{
RIFLESSIONI IN TEMA DI COMPARAZIONE, DIRITTO TRANSNAZIONALE E LEX SPORTIVA
}

\author{
Nicola Cavallaro \\ Avvocato nel Foro di Bergamo
}

\begin{abstract}
Con questo articolo si intende svolgere una riflessione sul grado di autonomia della lex sportiva sovranazionale rispetto agli altri sistemi di norme, prendendo in considerazione tre fattispecie che hanno suscitato molto dibattito nella prassi: il divieto di promozione pubblicitaria dei prodotti di tabacco ed assimilati in occasione di manifestazioni sportive, il problema dei diritti audiovisivi in relazione ad eventi sportivi e la fattispecie della c.d. Third Party Ownership nel rapporto tra normativa FIFA e quella della Unione Europea in tema di concorrenza.

In alcuni casi, come nel conflitto tra UEFA e FIFA da un lato ed Unione Europea dall'altro, sulla gestione dei diritti televisivi in ambito sportivo, è intervenuta la giurisprudenza della Corte di Giustizia dell'Unione Europea a risolvere la questione. In altri casi, si vuole mettere in rilievo come il conflitto in realtà si ponga solo come potenziale, ovvero apparente qualora si adotti un approccio rispettoso degli ambiti propri dei diversi sistemi di norme.
\end{abstract}

Keywords: Immunità, Pubblicità, Emittente, Concorrenza, Trasparenza

\begin{abstract}
This article intends to analyze the level of autonomy of the supranational sports rules compared to other systems of rules, and taking into consideration three cases that have aroused a great debate in practice: the prohibition of advertising promotion of tobacco products and similar in sports events, the problem of audiovisual rights in relation to sports events and the case of the so - called Third Party Ownership in the relationship between FIFA rules and the European Union regulations on competition matters. In some cases, such as in the conflict between UEFA and FIFA, on the one hand, and the European Union, on the other, on the management of television rights in sports, the jurisprudence of the European Court of Justice has intervened to resolve the conflict. In other cases, we want to emphasize how the conflict actually arises only as potential or apparent if you adopt an approach that respects the specific areas of the different systems of norms.
\end{abstract}

Keywords: Immunity, Advertising, Broadcaster, Competition, Transparency

\section{La crescente dimensione transazionale del diritto sportivo e la sua immunità}

L'approccio alla comparazione tra i vari ordinamenti giuridici risente ormai da tempo dell'esigenza di una rimodulazione in funzione dell'affermarsi della prospettiva transnazionale del diritto. Come è stato osservato da autorevole dottrina, "In a general sense, 'transnational law' may refer to any law which transcends state laws. It is therefore different from plain domestic laws, but it may include regional and international laws [...] Transnational 
law also has a different focus than traditional international law since its main concern is not international treaties and conventions which emanate from sovereign states". ${ }^{1}$ In particolare, si individuano diverse ipotesi di diritto transnazionale che possono configurarsi a livello privatistico o pubblicistico. Dal primo punto di vista, vengono in rilievo, ad esempio, contratti tra imprese che hanno sede in differenti Paesi. Con riguardo all'aspetto pubblicistico, si ricorda, tra l'altro, che gli Stati e le organizzazioni intergovernative possono coordinare sistemi di norme che hanno una dimensione transnazionale, il tutto anche su base informale. Per esempio, essi possono accordarsi su raccomandazioni che siano destinate ai legislatori dei vari Paesi, ai Giudici, agli individui o alle imprese oppure possono stabilire accordi in materia di cooperazione circa la applicazione transazionale di una legge, come in materia di cross-border law enforcement. ${ }^{2}$ La dottrina distingue poi - sempre nell'ambito di norme sovranazionali - tra 'international sports law' e 'global sports law', quest'ultima caratterizzandosi per una 'immunita' ovvero indipendenza e 'non condizionamento' da parte del diritto statale, ${ }^{4}$ impregiudicata la questione interna ai vari Stati e relativa ai rapporti tra ordinamento giuridico sportivo e quello statale. ${ }^{5} \mathrm{Si}$ osserva nello specifico come "Inside the sports world, private acts acquire a universal power such as the Olympic Charter. These sports rules claim immunity from national law and national legal proceedings". ${ }^{6} \mathrm{Ne}$ deriva che un approccio comparatistico soltanto a livello di confronto tra norme statali sembra risultare oggi insufficiente. ${ }^{7}$ Altri autorevoli Autori, ${ }^{8}$ inoltre, non pongono più ormai il criterio tradizionale delle "famiglie giuridiche" dei vari ordinamenti statali alla base del metodo comparativo, invocandosi altri modelli in funzione di quest'ultimo. Orbene, un ordinamento transnazionale ${ }^{9}$ si rivela in diverse aree del diritto, in particolare nel diritto commerciale, ma una prospettiva transnazionale si rinviene ormai anche in altri settori, tra cui il diritto dello sport e specialmente, come è stato osservato, in quelle materie sportive di rilevanza economica (Business Law). ${ }^{10}$ Accadono peraltro casi di conflittualità, o comunque di confronto, in cui la lex sportiva o l'istanza sportiva sovranazionale non riesce ad

${ }^{1}$ M. Siems, Comparative Law, From Transational Law to Global Law, 2nd ed., Cambridge, Cambridge University Press, 2018 , p. 307.
${ }^{2}$ Ivi, pp. 307-308.
${ }^{3}$ Sulla globalizzazione del diritto, mi si permetta di rinviare a N. Cavallaro, Considerations for a Global Private Law, in Law Journal of Thammasat University, vol. 42, 1, 2013.

${ }^{4}$ Cfr. K. Foster, Is There a Global Sports Law?, in The Entertainment and Sports Law Journal, vol. 2, 1, 2003 , p. 1 ss. Secondo cui "International sports law can be applied by national courts. Global sports law by contrast implies a claim of immunity from national law. Conceptually, it is a cloak for continued self-regulation by international sports federations and a claim for non-intervention by national legal systems and by international sports law" (p. 1 ss.).

${ }^{5}$ Sul punto, P. D’Onofrio, Corte costituzionale e ordinamento sportivo: reale autonomia?, in Diritto dello Sport, 4, 2016 , p. 267 e ss.

${ }^{6}$ M. Papaloukas, Lex sportiva and lex mercatoria, in International Sports Law Review Pandektis, vol. 10, 1-2, 2013, p. 202 ss. Secondo cui "Sports rules as they are globally applicable, they seem to affect the entire (sports) world" (p. 202 ss.).

${ }^{7}$ Cfr. M. Siems, Comparative Law, cit., p. 307. L'Autore rileva inoltre che: "Still, transnational law is a broad category that includes various types of legal rules. For example, writing about transnational commercial law, Roy Goode defines it as rules, from whatever source, which govern international commercial transactions and which are common to at least a significant number of legal systems, including, for instance, unwritten customs, contractually incorporated rules and trade terms promulgated by international organisations, standard term contracts and restatements of scholars" (ibidem, il riferimento è a R. Goode, in R. Goode, H. Kronke, E. McKendrick, Transnational Commercial Law: Text, Cases, and Materials, 2nd ed., Oxford, Oxford University Press, 2015, par. 1.02).

${ }^{8}$ Così Sir Basil Markesinis, il quale evidenzia che: "Any account of textbooks, treatises, or influential monographs of that period must start with France and René David's Les Grands Systèmes de droit contemporains [...] David's book suffered from a number of defects, some detectable when the work first appeared in print but all magnified with the passage of time [...] the classification of legal systems into more or less recognisable legal families can no longer easily accommodate a fascinating and (relatively) recent trend. This is the mutation of some systems which have deviated, or even departed, from their genealogical ancestors and moved, partially or more substantially, in the direction of 'rival systems'. Portugal and the Netherlands offer examples of systems which have moved away from their Roman and - later - French origins, and have either turned towards Germanic notions or created their own (mixed) systems, impregnated by Germanic concepts and the ideas found in German literature" (B. Markesinis, in B. Markesinis, J. Fedtke, Engaging with Foreign Law, Oxford-Portland, Oregon, Hart Publishing, 2009, pp. 4-5).

${ }^{9}$ Sugli aspetti transnazionali del diritto, si rinvia a A. Duval, What Lex Sportiva Tells You About Transnational Law, in P. Zumbansen (ed.), The many lives of transnational law. Critical engagements with Jessup's bold proposal, Cambridge , Cambridge University Press, 2019, il quale - nell'analizzare le diversità di utilizzo della nozione di diritto transnazionale nel linguaggio scolastico - concentra l'attenzione sulla lex sportiva come idoneo settore empirico dove potere studiare in concreto il concetto di transnazionalità: "Indeed, the regulation of international sports is nowadays often referred to as lex sportiva and deemed an example of transnational law" (ibidem).

${ }^{10}$ Cfr. M. Siems, Comparative Law, cit., pp. 305-306. 
affermare, quanto meno pienamente, quella propria 'immunità' dalle norme statali o da altri ordinamenti. Ciò si è verificato, in particolare, in materia di diritti televisivi in relazione ad eventi sportivi, nonché in tema di Consumer Law - con riguardo alla promozione pubblicitaria di prodotti dannosi (o comunque potenzialmente dannosi) per la salute del consumatore in occasione di manifestazioni sportive - e, infine, in relazione a profili di Competition Law coinvolti dalla applicazione di una norma sportiva sovranazionale.

\section{Il conflitto tra FIFA e UEFA, da una parte ed Unione Europea, dall'altra, in materia di diritti televisivi in relazione ad eventi sportivi}

Nell'ambito della disciplina dei diritti televisivi appena citata, si ricorda il conflitto tra UEFA (e FIFA), da una parte, ed Unione Europea, dall'altra, nato a seguito della emanazione della direttiva 89/552/CEE del Consiglio, che ha poi visto successive integrazioni ed aggiornamenti ${ }^{11}$, avente ad oggetto il coordinamento di disposizioni legislative, regolamentari e amministrative degli Stati membri concernenti l'esercizio delle attività televisive (c.d. direttiva "sulla televisione senza frontiere"), e la quale assegnò alle singole Autorità nazionali la facoltà di individuare determinati eventi da trasmettere obbligatoriamente anche su canali liberamente accessibili, affinché fosse garantita la possibilità per la stragrande maggioranza dell'utenza televisiva di goderne la visione. La FIFA e l'UEFA, organizzatrici rispettivamente delle fasi finali dei campionati mondiali ed europei di calcio, avevano tentato "di tutelare i contratti di pay-per-view firmati in esclusiva per la trasmissione di dette competizioni e costituenti la principale, ed indubbiamente considerevole, fonte dei loro redditi, contestando il diritto riconosciuto dall'Unione Europea ad ogni Stato membro di compilare un elenco di partite di particolare rilevanza per la società, necessariamente libere da qualsivoglia esclusiva, al fine di non privare una parte importante del pubblico della possibilità di seguire le medesime". ${ }^{12}$ Come è noto, è poi prevalso l'ordinamento comunitario, nella sua applicazione da parte della Corte di Giustizia della Unione Europea, la quale, rigettando le istanze della UEFA, ha ritenuto conformi e compatibili con la normativa europea (in relazione agli articoli (già) $49 \mathrm{CE}$ e $86 \mathrm{CE}$ ) le misure adottate da alcuni Stati membri sulla base di quella direttiva. ${ }^{13}$ In particolare, la giurisprudenza comunitaria ha escluso la sussistenza di una violazione dell'articolo 86 del Trattato CE citato (oggi articolo 106 TFUE), il quale vieta agli Stati membri di adottare misure - in favore di imprese pubbliche o con diritti speciali o in esclusiva che possano risultare in contrasto con le norme sulla concorrenza ai sensi delle disposizioni comunitarie.

Peraltro, il rispetto delle regole a tutela della concorrenza ovvero l'esigenza di prevenire situazioni di privilegio lesive del mercato oppure di monopolio costituisce - sempre nella materia della assegnazione di diritti audiovisivi in materia sportiva - un trend transnazionale sotteso alle norme di vari ordinamenti, volendosi, in particolare, evitare una concentrazione di tali diritti in capo ad un solo soggetto. In Italia, come è noto, la materia dell'aggiudicazione dei diritti audiovisivi in questione ha ricevuto una sistemazione organica in base al quadro normativo di cui al decreto legislativo Melandri/Gentiloni (d.lgs. n. 9/2008). L'art. 8 del d.lgs. n. 9/2008 ha

\footnotetext{
${ }^{11}$ In particolare, tale direttiva è oggi sostituita dalla Audiovisual Media Services Directive (2010/13/EU) la quale, tra l'altro, con riguardo all'ambito del divieto di promozione pubblicitaria e di sponsorizzazione del tabacco, di cui si dirà in seguito, estende la applicazione di tale divieto a tutte le forme di comunicazione commerciale audiovisiva.

${ }^{12}$ P. Pacileo, La vendita dei diritti televisivi di eventi sportivi: recenti sviluppi e spunti comparatistici, www.comparazionedirittocivile.it, 2013/2014, p. 1 e ss. L'Autore ricorda la pronuncia del 18 luglio 2013 della Corte di Giustizia dell'Unione Europea con la quale fu respinta "l'impugnazione avanzata dall'UEFA (Union European Football Associations) con cui si chiedeva l'annullamento della sentenza del Tribunale dell'Unione Europea del 17 febbraio 2011 che, a sua volta, aveva rigettato la domanda di annullamento parziale della decisione 2007/730/CE della Commissione, del 16 ottobre 2007, sulla compatibilità con il diritto comunitario delle misure adottate da Belgio e Regno Unito a norma dell'articolo 3 bis, paragrafo 1, della direttiva 89/552/CEE del Consiglio, avente ad oggetto il coordinamento di determinate disposizioni legislative, regolamentari e amministrative degli Stati membri concernenti l'esercizio delle attività televisive.

Dunque, in forza di tale sentenza, le autorità nazionali degli Stati membri hanno il diritto di vietare l'esclusiva televisiva per eventi di "particolare rilevanza sociale" e possono esigere che questi ultimi (ad esempio, i mondiali e gli europei di calcio) siano trasmessi su canali liberamente accessibili, per garantire il diritto del pubblico a vederli; d'altronde, ogni ampliamento delle facoltà connesse al diritto all'informazione comporta una restrizione delle potenzialità economiche derivanti dalla cessione dei diritti audiovisivi sull'evento" (ibidem).

${ }^{13}$ Cfr. ibidem.
} 
disciplinato l'assegnazione di tali diritti con il fine di favorire un esito in cui non vi sia, per l'appunto, un unico "vincitore" che possa disporre della intera offerta. Infatti, l'organizzatore della competizione "è tenuto ad offrire i diritti audiovisivi mediante più procedure competitive, ai fini dell'esercizio degli stessi per singola piattaforma ovvero mettendo in concorrenza le diverse piattaforme, ovvero con entrambe le modalità. Nell'ipotesi in cui vengano messe in concorrenza diverse piattaforme, l'organizzatore della competizione è tenuto a predisporre più pacchetti. L'organizzatore della competizione deve predisporre pacchetti tra loro equilibrati in modo da garantire la presenza, in ciascuno di essi, di eventi della competizione di elevato interesse per gli utenti". L'art. 9, comma 4, d.lgs. n. 9/2008, inoltre, ha previsto la c.d. "no single buyer obligation" secondo cui è "fatto divieto a chiunque di acquisire in esclusiva tutti i pacchetti relativi alle dirette, fermi restando i divieti previsti in materia di formazione di posizioni dominanti"; infine, l'art. 10, comma 1, d.lgs. n. 9/2008 ha disposto che i contratti di licenza abbiano una durata massima di tre anni. ${ }^{14}$

Se poi prendiamo in considerazione il mondo anglosassone, si osserva che anche negli Stati Uniti, circa la materia dei diritti televisivi sportivi, la normativa intende prevenire situazioni di chiusura del mercato. Infatti, i c.d. "broadcasters" investono al fine di ottenere il diritto di trasmettere eventi sportivi. Il concorrente vincitore la gara (di solito) ottiene la esclusività, tuttavia è prevista una apposita disciplina per la ipotesi in cui altri competitors siano in grado di intercettare e ritrasmettere i segnali. Cosi, ad esempio, altre compagnie emittenti possono intervenire in c.d. "passive retransmission", 15 dietro il versamento di un prezzo ("statutorily mandated fee to the Copyright Tribunal" ${ }^{\text {16 }}$ ), organo, quest'ultimo, che a propria volta redistribuisce in proporzione ai titolari dei diritti di copyright gli introiti ricevuti. ${ }^{17}$

Si individua, pertanto, in materia un orientamento transnazionale volto a garantire la presenza di più soggetti nel mercato.

\section{Il divieto di promozione pubblicitaria del tabacco e di prodotti assimilati in occasione di eventi sportivi: un caso di coesistenza di norme sportive sovranazionali con altre norme internazionali o domestiche}

Con riguardo alla pubblicità e alla promozione di prodotti del tabacco, si ricorda che - secondo la Direttiva 2003/33/CE del Parlamento Europeo e del Consiglio del 26 maggio 2003 (sul ravvicinamento delle disposizioni legislative, regolamentari e amministrative degli Stati membri in materia di pubblicità e di sponsorizzazione a favore di prodotti del tabacco) - sono vietate, all'interno degli Stati membri, varie forme di informazione, promozione e di pubblicità relative a tali prodotti, inclusa la sponsorizzazione connessa al tabacco, come pure la distribuzione gratuita dei medesimi, in relazione ad eventi (anche sportivi) ovvero ad attività che coinvolgano o abbiano luogo in vari Stati membri (o che producano in qualunque altro modo effetti transfrontalieri). ${ }^{18} \mathrm{~A}$ tal proposito, si osserva che alcune federazioni sportive internazionali avevano già deliberato - ben prima di tale direttiva - un divieto di sponsorizzazione del tabacco in occasione di eventi ovvero attività sportive (peraltro, la direttiva 2014 / 40 / UE del Parlamento Europeo e del Consiglio ha poi esteso alle sigarette elettroniche le

\footnotetext{
${ }^{14} \mathrm{Si}$ è riportato espressamente il quadro riassuntivo esposto in materia da F. Ghezzi, M. Maggiolino, La cessione dei diritti audiovisivi del Campionato di calcio di Serie A, tra regolazione procompetitiva e tutela della concorrenza, in Rivista della Regolazione dei Mercati, 2 , 2014, par. 1.

${ }^{15}$ Cfr. R.C. Berry, G.M. Wong, Law and Business of the Sports Industries, Common Issues in Amateur and Professional Sports, Volume II, Second edition, Praeger, Westport, Connecticut, 1993, 11.23.

${ }^{16}$ Ibidem.

${ }^{17}$ Ibidem. In proposito, ringrazio Prof. Glenn M. Wong, Executive Director, Sports Law \& Business Program, Distinguished Professor of Practice - Sports Law - Sandra Day O' Connor College of Law - Arizona State University e Mr Sam Renaut, Director, Sports Law \& Business Program, Arizona State University - Sandra Day O’Connor College of Law (111 E Taylor St, Phoenix, Arizona 85004) per avermi accolto alla Arizona State University nel mese di ottobre, 2018. In un colloquio con Mr Sam Renaut, egli sottolineò come negli Stati Uniti l'attività di vendita biglietti (oltre, si intende, a quella relativa agli introiti dai media, ai diritti di broadcasting e ai contratti di sponsorizzazione) rappresenti ancora una rilevante entrata economica nello sport.

${ }^{18}$ Peraltro, tale direttiva non riguarda la restrizione della promozione pubblicitaria e della sponsorizzazione del tabacco per il tramite della televisione, in relazione alla quale la disciplina si rinviene nella già citata Audio-visual Media Services Directive (2010/13/EU), mentre la Council Recommendation (2003/54/EC) on the prevention of smoking and on initiatives to improve tobacco control copre altre forme di promozione del tabacco.
} 
restrizioni in materia di pubblicità ovvero quelle in tema di comunicazioni commerciali audiovisive di cui alla direttiva 2010 / 13 / UE del medesimo Parlamento Europeo e del Consiglio (art. 20, comma 5, lett. e), ed ha anche previsto divieti ed obblighi di registrazione per le vendite transfrontaliere (art. 18). In particolare, la FIA (Federazione Internazionale dell'Automobile) nel 2001 decise per il c.d. "tobacco ban", concedendo alle scuderie cinque stagioni di tempo per rimuovere le marche di sigarette dalle carrozzerie. ${ }^{19}$ Peraltro, tale divieto viene interpretato in maniera più o meno stringente a seconda dello Stato ovvero dell'ordinamento del Paese in cui si verifica l'evento. Per esempio, in un recente Gran Premio di Formula 1 in Australia, la Ferrari non ha potuto riportare, sulle proprie monoposto e sulle tute di piloti e staff, il logo "Mission Winnow" perché le Autorità statali hanno ipotizzato una violazione delle norme contro la pubblicità del tabacco, considerato che "Mission Winnow" rappresentava una partnership tra Philip Morris International, Scuderia Ferrari e Ducati Corse istituita per promuovere le nuove sigarette a tabacco riscaldato. ${ }^{20}$ Ciò in quanto l'ordinamento australiano accoglie un divieto molto rigoroso in tema di promozione e pubblicità di prodotti del tabacco. Come è stato osservato, "Under Australian law it is illegal to advertise tobacco products on radio, television or in the print media. The impact of this is to effectively render impossible any marketing by tobacco companies in association with sport in any way", ${ }^{21}$ anche se la normativa (The Tobacco Advertising Prohibition Act 1992 (Cth)) non proibisce la sponsorizzazione in sé ma solo la promozione pubblicitaria del tabacco. ${ }^{22}$

In tale materia, si assiste pertanto ad una ipotesi in cui la lex sportiva sovranazionale coesiste con analoghe disposizioni di altri ordinamenti nazionali o pure sovranazionali ovvero persino anticipa tali analoghe disposizioni, cosi come sopra visto con riguardo alla decisione della FIA del 2001 che anticipò la direttiva 2003/33/CE in materia di divieto di promozione pubblicitaria dei prodotti del tabacco per eventi transfrontalieri.

\section{Un caso di apparente conflitto: il divieto previsto dalle norme FIFA con riguardo alla c.d "Third Party Ownership" (“TPO") e la questione della sua compatibilità con la normativa dell'Unione Europea a tutela della concorrenza}

In altri casi ancora si assiste ad una tensione caratterizzata da conflittualità tra lex sportiva sovranazionale ed altri ordinamenti, dovendo invece chiedersi - preliminarmente e prima di potere avanzare ipotesi di contrasto - se gli ambiti ed i limiti spettanti per materia ai diversi sistemi di norme siano stati rispettati. Così, in tema di diritto

\footnotetext{
${ }^{19}$ Sul punto: https://www.automoto.it/formula1/2007-2017-10-anni-di-formula-1-senza-tabacco.html.

${ }^{20}$ Sul punto: https://tobaccoendgame.it/news/la-pubblicita-del-tabacco-torna-nel-campionato-di-formula-1/

${ }^{21}$ D. Thorpe et al., Sports Law, Second Edition, Oxford University Press Australia \& New Zealand, 2013, p. 504. Gli Autori fanno anche riferimento, sul punto, a R. Furlong, Tobacco Advertising Legislation and the Sponsorship of Sport, in Australian Business Law Review, 22 (3), 1994, p. 159.
}

${ }^{22}$ Cfr. D. Thorpe et al., Sports Law, cit., pp. 504 e ss. Gli Autori ricordano come "The Tobacco Advertising Prohibition Act 1992 (Cth) does allow incidental appearance of tobacco products in a broadcast or publication, provided there is no benefit to the broadcaster or publisher. However, there is no prohibition on sponsorship per se; it is just a matter of no advertising being permitted. Hence a tobacco sponsorship in Sport may be acknowledged, but that must be done within the limits of the Act. Breach of the Act will lead to severe financial penalties and possible loss of license under the Broadcasting Services Act 1992 (Cth)" (ibidem, p. 504). Gli stessi Autori, inoltre, con riguardo al tema dell'alcool, così riassumono la disciplina in Australia: "On the other hand, the marketing of alcohol with sport is only minimally regulated. Sponsorship of sport by alcohol-related companies is without any regulation, while the advertising of alcohol generally is 'self-regulated' [citando, in proposito, M Mallam, A Commentary on Two of Australia's Greatest Consuming Passions, Alcohol and Sport, and the Regulation of the Relationship Between Them, in ANZSLJ, 1 (1), 2006, p. 42. ndr]. Regulation is managed by the Advertising Standards Bureau; however the limitations on marketing are of no significant consequence to alcohol promotion in connection with sport. A voluntary regulatory code and vetting system of alcohol advertising standards is operated under the Alcoholic Beverages Advertising Code (ABAC), which is formulated by representatives of the Australian Government, the Advertising Federation of Australia and the leading alcohol beverage industry associations. Under ABAC all advertisements for alcohol beverages produced for publication or broadcast in Australia may not: a) suggest that the consumption or presence of alcohol beverages may create or contribute to a significant change in mood or environment or depict the consumption or presence of alcohol beverages as a clause of contributing to the achievement of sporting success; or b) depict any direct association between the consumptions of alcohol beverages, other than low alcohol beverages and the engagement in any sport activity. The ABAC does not cover sport product names and packaging or sponsorship. In terms of penalty for breach of the code, this seems to be limited to the removal of advertisement" (ivi, pp. 504-505). 
della concorrenza, è noto che il divieto di "Third Party Ownership"23 (“TPO”), di cui al corrente articolo 18-ter ${ }^{24}$ del Regolamento FIFA ("Fédération Internationale de Football Association") sullo status e sui trasferimenti dei calciatori, è stato, ed è tuttora, oggetto di controversie giurisprudenziali e di ampio dibattito dottrinale in relazione alla sua applicazione, in particolare proprio sotto il profilo del rispetto della normativa della Unione Europea in tema di concorrenza; recenti pronunce giurisprudenziali nazionali (caso Seraing ${ }^{25}$ ), dovendo trattare di fattispecie relativa a tale materia, hanno persino sancito in via preliminare l'illegittimità delle clausole arbitrali contenute negli statuti della FIFA e della UEFA ("Union of European Football Associations") di attribuzione della giurisdizione al Tribunale Arbitrale dello Sport (con divieto generale ed assoluto di ricorso al Giudice Ordinario) in quanto ritenute contrarie, tra l'altro, ai principi sanciti dall'articolo 6 della Convenzione Europea dei Diritti dell'Uomo ("Diritto a un equo processo") e dall'articolo 47 della Carta dei Diritti Fondamentali dell'Unione Europea ("Diritto a un ricorso effettivo e a un giudice imparziale").

Per "TPO" si intende quell'istituto, come è stato definito in senso ampio ${ }^{26}$, in virtù del quale un terzo soggetto finanzia lo sviluppo ("development") ovvero l'incremento di valore professionale di un giocatore, ricevendo quale corrispettivo il riconoscimento dei diritti relativi al futuro valore di trasferimento di quel giocatore; mentre, in alcuni casi, il terzo soggetto investitore viene anche "utilizzato" come metodo di finanziamento per acquistare giocatori in favore di club che non hanno sufficiente denaro per finanziare i rispettivi affari per conto proprio ("on their own"), il tutto per il tramite dell'acquisto di parte o della intera titolarità dei diritti economici relativi a quel dato giocatore, cosicché il terzo soggetto investitore riceverà tutto o parte del valore di trasferimento ("transfer value") del giocatore stesso.

Orbene, fatta questa premessa, si rileva che gli sforzi di inquadramento e di studio, sinora compiuti, sono andati nella direzione di analizzare ed accertare se il divieto di "TPO", di cui alle norme FIFA, sia o meno compatibile con il diritto dell'Unione Europea, in particolare con la normativa europea a tutela della concorrenza (articoli 101 e ss. TFUE). In proposito, ed in sintesi, si è pervenuti a posizioni, vuoi contrarie vuoi a favore, a seconda che si valorizzino, tra le opposte istanze invocate più di recente, la c.d. massimizzazione del consumer welfare $e^{27}$ da un lato $^{28}$ oppure la necessità di trasparenza della governance nel calcio, dall'altro lato, ovvero la esigenza di avere informazioni univoche su chi sia effettivamente titolare dei diritti economici dei calciatori, a tutela del buon andamento del mercato. ${ }^{29} \mathrm{Si}$ invoca anche, proprio in vista di tale tutela, e a favore del divieto di "TPO", il rispetto del c.d. fair play finanziario (anche sotto il profilo della pubblicità circa la reale capacità economica dei club beneficiari delle prestazioni dei giocatori), invocandosi la tutela della stabilità finanziaria delle società sportive. ${ }^{30}$

${ }^{23}$ Sul tema della c.d. "Third Party Ownership", mi si permetta di rinviare a N. Cavallaro, La Third Party Ownership: riflessioni di ammissibilità nel nostro ordinamento, in Diritto dello Sport, 3-4, 2013, pp. 155 e ss.

${ }^{24}$ Articolo 18-ter ("Third-party ownership of players'economic rights") del Regolamento FIFA sullo status e sui trasferimenti dei calciatori (comma 1): "No club or player shall enter into an agreement with a third party whereby a third party is being entitled to participate, either in full or in part, in compensation payable in relation to the future transfer of a player from one club to another, or is being assigned any rights in relation to a future transfer or transfer compensation".

${ }^{25} \mathrm{Cfr}$. Corte d'Appello Civile di Bruxelles 6348/2018. Sul punto, per i commenti della stampa, si rinvia a: https://www.panorama.it/economia/seraing-tas-losanna-corte-appello-bruxelles.

${ }^{26}$ Cfr. L. Villas, B. Pires in Third party ownership - to ban or not to ban?, in LawInSport, 10.12.2013. Sempre sul tema, N. Cavallaro, La Third Party Ownership, cit.

${ }^{27}$ Cfr. R. Whish, D. Bailey, Competition Law, Seventh ed., Oxford University Press, 2012, p. 4. Sul tema mi si permetta di rinviare anche a N. Cavallaro, Riflessioni in tema di tutela della concorrenza in Unione Europea, fondi di investimento e sport, in Diritto dello Sport, 4 , 2016, pp. 221 e ss.

${ }^{28} \mathrm{Col}$ rischio - se non si consentono investimenti - di contrarre la frequenza dell'acquisto del biglietto per la visione di partite allo stadio ovvero la sottoscrizione di programmi e canali TV e satellitari che abbiano l'esclusiva dei diritti di trasmissione di quella data società sportiva, ma anche in virtù di un minore acquisto di prodotti in merchandising sportivo.

${ }^{29}$ Cfr. R. Andrews, Third Party Investment, in EPFL Sports Law Bulletin, 10th Edition, 2012, p. 34.

${ }^{30}$ A tale ultimo proposito, si ricorda che "Lo strumento predisposto in ambito UEFA per soddisfare l'esigenza di tutelare la stabilità finanziaria del settore calcio prende il nome di Financial Fair Play (FFP). Esso fa riferimento ad un insieme di regole, entrate in vigore nel 2012 per opera del Comitato Esecutivo UEFA e dei rappresentanti dei club, predisposte con l'obiettivo di tutelare la sostenibilità finanziaria del movimento calcistico europeo. Il FPP nasce dunque dal bisogno di fronteggiare un problema, quello dell'indebitamento, che è diventato assolutamente attuale nel settore calcistico. Infatti, molte delle più blasonate squadre europee mostravano, al momento 
Orbene, onde affrontare tale tema, occorre in primo luogo ricordare quali siano gli interessi protetti ovvero gli obiettivi della normativa UE in tema di concorrenza. La dottrina maggioritaria riconosce che l'obiettivo generale della normativa a tutela della libera concorrenza sia quello di tutelare ovvero "maximise consumer welfare", il che comporta che il gioco della concorrenza consente l'ingresso di vari operatori in competizione tra di loro al fine di produrre e distribuire prodotti e servizi sempre più innovativi e qualitativamente migliori ed efficienti, anche con produzione degli stessi a prezzi sempre più convenienti, il tutto a tutela del consumatore, che dispone di una ampia libertà di scelta. ${ }^{31} \mathrm{D}$ 'altra parte, al di là di questo scopo generale, ed a margine degli altri profili (rispetto ai quali si rinvia alla letteratura in materia ${ }^{32}$ ), la politica di concorrenza dell'Unione Europea persegue anche un altro obiettivo specifico che è quello della creazione e del rafforzamento del mercato interno. A tal proposito, sempre come sottolineato dalla migliore dottrina, il diritto della concorrenza dell'Unione ha anche un ruolo da svolgere in funzione di tale integrazione del mercato unico in quanto "it can prevent measures which attempt to maintain the isolation of one domestic market from another: for example national cartels, export bans and market-sharing will be seriously punished". ${ }^{33} \mathrm{Ne}$ risulta che ogni accordo o pratica che contribuisca all'isolamento del mercato o che pregiudichi la ottimizzazione della scelta da parte dei consumatori, ovvero che determini barriere all'ingresso di altri operatori, si pone in contrasto con la politica e la normativa in tema di concorrenza dell'Unione Europea. In secondo luogo, occorre ricordare la ampiezza riconosciuta alla portata applicativa della disposizione di cui all'articolo 101 TFUE, nell'ambito delle regole di concorrenza previste dal Trattato in questione, in quanto secondo tale norma sono vietati tutti gli accordi, le decisioni ed in generale tutte le forme di pratiche concordate, che possano pregiudicare il commercio tra Stati membri e che abbiano per oggetto ma anche per effetto di impedire, restringere o falsare il gioco della concorrenza all'interno del mercato interno. Ciò significa che, anche se l'esame dell'oggetto di tali atti o comportamenti potesse portare in un primo momento a non ritenere gli stessi illeciti, occorrerà poi tuttavia analizzare se, a prescindere dall'oggetto, vi sia comunque come effetto quello di incidere negativamente nel gioco della concorrenza. A tal proposito, la giurisprudenza della Corte di Giustizia dell'Unione Europea ha chiarito, sotto il profilo dell'oggetto, che "per valutare se un accordo tra imprese o una decisione di associazione di imprese presentano un grado sufficiente di dannosità per essere considerati come una restrizione della concorrenza "per oggetto" ai sensi dell'articolo 81, paragrafo 1, CE, occorre riferirsi al tenore delle loro disposizioni, agli obiettivi che essi mirano a raggiungere, nonché al contesto economico e giuridico nel quale essi si collocano. Nella valutazione di tale contesto, occorre prendere in considerazione anche la natura dei beni o dei servizi coinvolti e le condizioni reali del funzionamento e della struttura del mercato o dei mercati in questione" ${ }^{34}$ La stessa giurisprudenza della Corte aggiunge, tuttavia, che quando la probabilità dannosa di certi comportamenti collusivi, quali quelli che portano alla fissazione orizzontale dei prezzi da parte di cartelli - con effetti negativi, in particolare, sul prezzo, sulla quantità o sulla qualità dei prodotti e dei servizi - sia "talmente alta" allora "può essere ritenuto inutile, ai fini dell'applicazione dell'articolo 81, paragrafo 1, CE, dimostrare che tali comportamenti hanno effetti concreti sul mercato", ${ }^{35}$ risultando pertanto non necessario ovvero superfluo un successivo esame in merito all' "effetto" negativo sulla concorrenza.

Orbene, fatta questa premessa illustrativa, risulta che un divieto di "TPO" se non per l'oggetto, quanto meno per l'effetto, sembra porsi non soltanto in violazione della massimizzazione del consumer welfare, ma altresì come barriera all'ingresso, ovvero al mantenimento, in particolare, di molti club che non dispongono di sufficienti

dell'entrata in vigore del FFP, significativi deficit di bilancio" (F. Trafficante, Profili critici e comparatistici dei sistemi salariali nello sport professionistico: due casi a confronto, in European Journal of Sport Studies (EJSS), 2014, punto 7.2). Lo stesso Autore ricorda poi che l'espressione "Financial Fair Play (FFP)" corrisponde, in realtà, ad una definizione di sintesi: "Più precisamente, UEFA Club Licensing and Financial Fair Play Regulations. Questa denominazione è giustificata dal fatto che questo insieme di regole va ad integrare e completare una disciplina già esistente, quella del c.d. License UEFA" (ibidem, nota 209). Sulla questione si veda anche: F. Casarola, TPO: un altro mostro da combattere per l'UEFA, in Iusport Italia, diritto e marketing sportivo, http://www.iusport.it/index.php?option=com_content\&view=article \&id=259\&catid=16\&Itemid=117.

${ }^{31}$ Cfr. R. Whish, D. Bailey, Competition Law, $7^{\mathrm{th}}$ ed., Oxford, Oxford University Press, 2012, p. 4.

${ }^{32}$ Ivi, pp. 4 e ss.

${ }^{33}$ Ivi, p. 23.

${ }^{34}$ Corte di Giustizia dell'Unione Europea, C- 67 / 13 P.

${ }^{35}$ Corte di Giustizia dell'Unione Europea, C- 67 / 13 P. 
risorse per finanziarsi on their own nell'attuale mercato e contesto sportivo. E' poi evidente, sotto il primo profilo del consumare welfare, che il divieto di "TPO" incide negativamente rispetto alla tutela delle scelte del consumatore-tifoso, il quale, di fronte a un $c l u b$ che non abbia idonei strumenti per finanziarsi, potrebbe essere indotto a ridurre la propria capacità di affezione e di determinazione di spesa in favore dello stesso $c l u b$, di cui è supporter, contraendo la frequenza dell'acquisto del biglietto per la visione di partite allo stadio ovvero la sottoscrizione di programmi ed abbonamenti di canali TV e satellitari che abbiano l'esclusiva dei diritti di trasmissione audiovisiva di quella data società sportiva, ma anche in virtù di un minore acquisto di prodotti in merchandising.

Sotto altro profilo, la normativa uniforme introdotta in sede FIFA con riguardo agli accordi di "TPO" ha risposto anche alla esigenza del controllo dei flussi finanziari e delle esposizioni debitorie ovvero a quella del rispetto del cosiddetto Fair-Play Finanzario di cui si espresso preciso orientamento in ambito UEFA. ${ }^{36} \mathrm{La}$ disciplina UEFA fa riferimento, infatti, nell'ambito dei propri undici "valori" di recente elaborati, alla integrità dello sport e alla regolarità delle competizioni anche sotto tale profilo in virtù del principio di cui al numero 8 intitolato "Fair Play Finanziario e regolarità delle competizioni"). ${ }^{37}$ A tale ultimo proposito, si ricorda che "Lo strumento predisposto in ambito UEFA per soddisfare l'esigenza di tutelare la stabilità finanziaria del settore calcio prende il nome di Financial Fair Play (FFP). Esso fa riferimento ad un insieme di regole, entrate in vigore nel 2012 per opera del Comitato Esecutivo UEFA e dei rappresentanti dei club, predisposte con l'obiettivo di tutelare la sostenibilità finanziaria del movimento calcistico europeo. Il FPP nasce dunque dal bisogno di fronteggiare un problema, quello dell'indebitamento, che è diventato assolutamente attuale nel settore calcistico. Infatti, molte delle più blasonate squadre europee mostravano, al momento dell'entrata in vigore del FFP, significativi deficit di bilancio". ${ }^{38}$ È evidente che il principio del pareggio di bilancio e di un "budgetary cap imposed on football clubs" sarebbe stato frustrato ove si fossero continuate ad ammettere esposizioni debitorie, spesso non trasparenti e senza alcun controllo, nei confronti di terzi soggetti. ${ }^{39}$ Il tutto a prescindere dal dibattito

${ }^{36}$ Cfr. F. Casarola, TPO: un altro mostro da combattere per l'UEFA, cit.

${ }^{37}$ Sul punto: http://it.uefa.org/about-uefa/eleven-values/index.html.

${ }^{38}$ F. Trafficante, Profili critici e comparatistici dei sistemi salariali nello sport professionistico: due casi a confronto, cit., punto 7.2 .

${ }^{39} \mathrm{Da}$ un altro punto di vista, si ha riguardo al tema dell'equilibrio della competizione e della salvaguardia della stabilità della stessa. Cosi, il controllo dei costi nel mondo dello sport, ed in particolare dei salari ovvero degli emolumenti garantiti agli sportivi, è pure in funzione della protezione della stabilità della competizione. Tale materia è ben presente nell'ordinamento sportivo statunitense. Come è stato osservato, "Alcune delle maggiori leghe professionistiche statunitensi hanno infatti introdotto, per mezzo dell'accordo collettivo (Collective Bargaining Agreements), regolamentazioni che fissano una corposa restrizione alla libertà contrattuale e finanziaria dei team coinvolti, ponendo di fatto un limite quantitativo (e qualitativo) alla possibilità di elargizione dei salari. Un sistema tale, che trova il suo fulcro nella disciplina del salary cap, risponde a due distinte esigenze: tutelare l'equilibrio competitivo nella competizione e salvaguardare la stabilità finanziaria delle squadre che vi partecipano": cosi F. Trafficante, Profili critici e comparatistici dei sistemi salariali nello sport professionistico: due casi a confronto, cit., sub introduzione. Senonché, in tale materia, occorre ricordare la problematicità di regolamentazioni che, seppure in funzione di coordinamento o di cooperazione, possono produrre come effetto un controllo sui prezzi (e sui salari) in quanto le stesse potrebbero essere intese in restrizione della concorrenza e, quindi, da analizzare sotto il profilo della normativa antitrust (in UE il problema si è posto con riguardo, tra l'altro, al rispetto dell'articolo 101 del Trattato sul Funzionamento dell'Unione Europea - TFUE - Si rinvia a: http://eur-lex.europa.eu/legalcontent/IT/TXT/?uri=CELEX:12012E/TXT), il tutto anche con riguardo alla formulazione del principio del competitive balance. Sulla base di tale principio (del c.d. "competitive balance") si fondano le seguenti idee cardine: "In primo luogo, la competizione fra le varie franchigie presuppone un'opera di coordinamento e cooperazione fra i vari concorrenti. In secondo luogo, in una Lega professionistica, i vari competitori non sono realmente interessati all'estromissione dei concorrenti dal mercato; infatti, perché possa esistere la competizione, è necessario che vi sia una pluralità di soggetti sportivi. In terzo luogo, detto principio genera l'idea di base per la quale più si tende all'equilibrio competitivo delle franchigie concorrenti ed all'incertezza del risultato (uncertain outcome hypothesis), più aumenta l'interesse del pubblico, e quindi il profitto economico (biglietti, merchandising etc)" (cosi, F. Trafficante, Profili critici e comparatistici dei sistemi salariali nello sport professionistico: due casi a confronto, cit., sub punto 6). Tale impostazione viene però criticata da una parte della dottrina e della giurisprudenza per avere forti implicazioni anti-concorrenziali. Su tale aspetto, e sulla dottrina e sulla giurisprudenza in merito al rapporto tra normativa antitrust dell'Unione Europea e ordinamento sportivo, si rinvia a S. Bastianon, Sport, antitrust ed equilibrio competitivo nel diritto dell'Unione europea, in Il Diritto dell'Unione Europea" 2012. Sulla applicabilità del diritto antitrust al settore dello sport, si veda il testo conclusivo della indagine conoscitiva IC27 riportato nel sito della Autorità Garante Concorrenza e del Mercato (www.agcm.it). Si veda anche D. Gullo, L'impatto del Diritto della Concorrenza sul 
tuttora in corso circa la compatibilità del medesimo Fair-Play Finanziario con la normativa dell'Unione Europea, in particolare di quella antitrust. ${ }^{40}$ Secondo parte della dottrina ${ }^{41}$ infatti, nonostante non sia ancora intervenuta una diretta pronuncia in materia da parte della Corte di Giustizia della Unione Europea, il Fair-Play Finanziario può qualificarsi come una decisione da parte di una associazione di imprese con effetto anticoncorrenziale ai sensi dell'art. 101 TFUE. Ciò in quanto, il Fair-Play Finanziario avrebbe come effetto quello di distorcere la concorrenza all'interno del mercato interno consistendo lo stesso, in particolare, in un accordo di fissazione indiretta dei prezzi tra compratori di beni e servizi: posto che i club competono tra loro nel mercato del calcio in quella 'materia prima' che sono i giocatori, dovendo adesso gli stessi adattare le proprie possibili spese in funzione delle entrate (in virtù del principio del pareggio di bilancio), viene meno quel libero gioco della concorrenza che altrimenti avrebbe luogo senza il Fair-Play Finanziario in questione. ${ }^{42}$

In realtà, tornando alla premessa, impostata cosi la questione (in termini di conflitto tra opposte istanze) si rischia di perdere di vista l'ambito della lex sportiva rispetto a quello proprio di altri sistemi. Che sport e business siano ormai sempre più collegati è manifesto, ma che i sistemi di norme sportive possano vietare attività economiche nuove o con profitto per terzi soggetti, come anche l'ingresso di nuovi soggetti investitori nello sport, non risulta tipico dei vari ordinamenti sportivi, se non quando siano in gioco diritti assoluti ed inviolabili della persona, come, ad esempio, il diritto alla salute (cosi, come sopra visto, per il c.d. "tobacco ban"). Pertanto, al di là dell'effetto negativo nella economia dello sport, per il quale limitare l'apporto di capitali esterni ai club di calcio in un periodo di crisi economica generalizzato comporterebbe oggi l'impossibilità, per molte società sportive, di finanziare il trasferimento dei calciatori da un $c l u b$ all'altro, un divieto di "TPO" sembrerebbe risultare non propriamente in sintonia con l'ambito specifico della lex sportiva, dovendo essere assoggettato, per come si evince da quanto sopra esposto, ad altri sistemi di norme, in primis alla normativa a tutela della concorrenza,

mondo dello sport (http://www.rdes.it/RDES_3_07_GULLO.pdf), intervento in occasione del convegno "Sport: Diritto, Fisco e Concorrenza", Roma, 21 settembre 2007.

${ }^{40}$ Come ricorda S. Bastianon (in Nessuno mi può giudicare... ovvero: il 'fair-play' finanziario e lo strano caso del signor Striani, in https://www.rivisteweb.it/doi/10.1434/82232) "La vicenda Striani rappresenta il primo caso nel quale è stata posta in discussione la compatibilità della regola Uefa sul pareggio di bilancio con il diritto antitrust dell’Unione europea. Sebbene né la Commissione, né il giudice nazionale, né la Corte di giustizia, per ragioni differenti, abbiano adottato una decisione nel merito, il caso Striani fornisce all'interprete un formidabile banco di prova per analizzare i diversi profili giuridici, di merito e processuali, emersi nelle varie fasi del giudizio". "The case received much attention on the part of the media, not only because Mr. Striani is represented by the very same lawyer of the groundbreaking Bosman ruling, Jean Louis Dupont, but also because almost all European football stakeholders are to some extent affected by the FFP. Most notably, above and beyond the obstacles to free movement rights and the possible violation of antitrust law, the respect of fundamental rights and the principle of non-discrimination can be questioned. [However, $n d r$ ], On July 16th, the Ninth Chamber of the ECJ issued an order declaring the above-mentioned reference for a preliminary ruling 'manifestement irrecevable" (R. Molé, The curious case of Daniel Striani (C-299/15): a missed opportunity, pubblicato il 21.09.2015, in http://rivista.eurojus.it/tag/fair-play-finanziario/). Si rileva, quindi, che, a causa della delibazione di manifesta irricevibilità della questione, la Corte di Giustizia dell'Unione Europea non ha, infine, esaminato la compatibilità del c.d. Fair-Play Finanziario con la normativa europea rispetto ai profili sopra citati.

${ }^{41}$ R. Molé, The curious case of Daniel Striani (C-299/15): a missed opportunity, cit.

${ }^{42}$ Ibidem. L'Autore prosegue il ragionamento evidenziando che, secondo la sua linea di pensiero, una tale decisione non può giustificarsi neppure ai sensi dell'art. 101, comma 3 TFUE perché i quattro sottocriteri ivi sanciti devono essere soddisfatti cumulativamente e il Fair-Play Finanziario non è affatto indispensabile perché si possono contemplare altre misure meno restrittive per raggiungere gli stessi obiettivi. Lo stesso Autore poi analizza anche la compatibilità con l'art. 102 TFUE invocando un abuso di posizione (super) dominante della UEFA, mettendo in rilievo che "UEFA itself is an undertaking, therefore it is necessary to delimit a relevant market where a dominant position can be assessed. In this regard, suffice it to consider the market for European-wide clubs competitions where the UEFA offers its product UEFA Champions League (UCL) to clubs, which are consumers thereof. The UCL is not interchangeable by consumers: the only other similar product is the UEFA Europa League (UEL), which is far less prestigious and profitable; the FFP applies to prospective participants of both the UCL and the UEL, therefore if one club could choose, it would go for the UCL straightaway. Plus, there is no rival product because the UEFA acts as monopolist. The geographic market being the whole EU territory, it is clear how the UEFA occupies a dominant position. So far so good, but: is there an abuse? I believe the answer to be yes. Indeed, a position such as UEFA's can be called of 'superdominance', where the duties towards the competition process are not merely negative but also positive. Not only is there no trace of positive duties towards all the consumers and the competition process itself, but also - for how the FFP is tailored - bigger clubs benefit to the detriment of the smaller ones. Thus, there is room for claiming an abuse of such a 'superdominant' position in the abovementioned relevant market. Moreover, the abuse cannot be justified, lacking proportionality to whatever reason might be put forward" 
anche europea; ed invocandosi, pertanto, una disciplina - nell'ambito di tale normativa appena citata - che stabilisca i limiti di ammissibilità dell'intervento di un terzo soggetto, con meri fini di investimento, in settori che riguardino attività economica ma che abbiano finalità e profili ulteriori ovvero più ampi, cosi come lo sport in relazione ai suoi aspetti sociali e ricreativi; ${ }^{43}$ e che consenta, quindi, di stabilire se e quando tali meri fini possano risultare compatibili con l'obiettivo generale delle disposizioni a tutela della concorrenza dato dalla massimizzazione del consumer welfare. ${ }^{44}$

\section{Conclusioni}

L'analisi delle fattispecie sportive oggi comporta la necessità di un approccio diverso ed ulteriore rispetto a quello tradizionale, relativo alla fondamentale questione della autonomia dell'ordinamento giuridico sportivo, in quanto lo sport è diventato socialmente ed economicamente sempre più significativo intervenendo anche sulla applicazione dei principi giuridici sottesi a tutte le materie dallo stesso coinvolte ("Sporting activity has contributed numerous developments to the application of general principles of law" ${ }^{\text {"45 }}$ ), avendo lo sport uno specifico impatto sul diritto sostanziale ("impact on substantive law") ${ }^{46}$, Bisogna però ricordare che rimane sempre un ambito tipico e proprio delle diverse discipline giuridiche, il cui rispetto può aiutare a prevenire ovvero a risolvere situazioni di contrasto facendo prevalere quel sistema di norme che per ratio sia maggiormente idoneo a regolare quella determinata fattispecie. Il diritto transnazionale, infatti, comprende vari tipi di norme giuridiche, che governano, ad esempio, le transazioni commerciali internazionali e che sono comuni ad un numero significativo di sistemi giuridici, ricordandosi, in proposito, le condizioni commerciali o i formulari di contratti standard elaborati dalle organizzazioni internazionali.

Con riguardo alle norme sportive transnazionali (global sports law), se si tiene conto che le stesse si caratterizzano, per come sopra visto in premessa, per una 'immunità' ovvero un 'non condizionamento' delle stesse da parte del diritto statale, ${ }^{47}$ si può concludere che il rispetto dell'ambito tipico e proprio della lex sportiva transnazionale, e il volere disciplinare, entro tale ambito, soltanto le tematiche tipicamente sportive, può aiutare a prevenire situazioni di conflitto con altri sistemi di norme.

\footnotetext{
${ }^{43} \mathrm{Cfr}$. A. Santuari, Le associazioni non profit operanti nel settore turistico-ricreativo e sportivo. Finalità perseguite e attività esercitate, in Diritto dello Sport, 3-4, 2013, pp. 164 e ss.

${ }^{44}$ Cfr. N. Cavallaro, Riflessioni in tema di tutela della concorrenza in Unione Europea, fondi di investimento e sport, cit., pp. $221 \mathrm{e} \mathrm{ss.}$

${ }^{45}$ D. Thorpe et al., Sports Law, cit.., pp. 1-2.

${ }^{46}$ Ibidem.

${ }^{47}$ Cfr. K. Foster, Is There a Global Sports Law?, cit., pp. 1 e ss. Come già ricordato, secondo tale Autore “International sports law can be applied by national courts. Global sports law by contrast implies a claim of immunity from national law. Conceptually, it is a cloak for continued self-regulation by international sports federations and a claim for non-intervention by national legal systems and by international sports law" (ibidem).
} 